\title{
A propósito de tres diccionarios de $\mathrm{DaF}$ : Sobre el Estado de la Lexicografía de Aprendices del Alemán
}

[Three German's Learner's dictionaries: State of the art of the German Learner's

\author{
Lexicography]
}

http://dx.doi.org/10.11606/1982-8837223667

\section{Félix Valentín Bugueño Miranda ${ }^{1}$}

A mi hermana Cristina Liliana Bugueño Miranda, que disfruta del alemán tanto como yo

\begin{abstract}
A learner's dictionary is a dictionary specially designed to help learners of a foreign language. In spite of a long tradition in lexicographical research, dictionaries for students of German language show quite a lack of development in regard to its Lexicography. The purpose of this paper is to evaluate the state of the art of German Pedagogical Lexicography by means of three learner's dictionaries. As a methodological framework we use metalexicographic principles, like the distinction between a quantitative and a qualitative macrostructural definition, the analysis of the microstructural information program and the distinction between decoding and encoding functions. As a result one may say that the three dictionaries are reliable language reference works for students of German as a Foreign Language.
\end{abstract}

Keywords: German as a Foreign Language; Lexicography; Lerner's dictionary.

Resumen: Un diccionario de aprendices es un diccionario especialmente diseñado para ayudar a aprendices en el proceso de enseñanza-aprendizaje de una lengua extranjera. A pesar de una larga tradición en investigación lexicográfica, los diccionarios de aprendices del alemán constituyen un desarrollo tardío de la lexicografía de esa lengua. El objetivo del presente trabajo es evaluar el estado del arte de la lexicografía pedagógica del alemán por medio de tres diccionarios de esa clase de obras lexicográficas. Como marco metodológico se emplean principios metalexicográficos tales como la distinción entre definición macroestructural cuantitativa y cualitativa, el análisis del programa informaciones microestructurales y la distinción entre funciones de decodificación y codificación. Así se establece que estos diccionarios son obras de referencia confiables para el aprendiz del alemán como lengua extranjera.

Palabras-clave: Alemán como Lengua Extranjera; Lexicografía; Diccionario de aprendices

\footnotetext{
${ }^{1}$ Universidade Federal do Rio Grande do Sul, Av. Bento Gonçalves, 9500, Porto Alegre, 91540000, Brasil. E-mail: felixv@uol.com.br
}

\section{(cc) BY-NC}




\section{Introducción}

Sin lugar a duda, el escenario lexicográfico alemán [Wörterbuchlandschaft] se ha enriquecido considerablemente con la consolidación de los diccionarios de aprendices en esa lengua ${ }^{2}$.

Según Rothenhöfer (2013: 414), los diccionarios de aprendices del alemán como lengua extranjera [DaF Lernerwörterbücher] constituyen un desarrollo muy tardío de la lexicografía alemana ${ }^{3}$ con relación al caso de la lexicografía inglesa. Este desarrollo tardío redunda en que la lexicografía de aprendices de alemán merezca una especial atención con el objetivo de evaluar la calidad de dichas obras.

El objetivo de este trabajo es evaluar tres diccionarios de aprendices de alemán, a fin de determinar qué reformulaciones estructurales se hacen necesarias en dichas obras. Una reformulación estructural es todo cambio necesario en los niveles macro-, micro- y/o medioestructurales. En Borba y Bugueño Miranda (2012: 34) se destaca que estos niveles son componentes canónicos de un diccionario de orientación semasiológica. Por ello, hay que considerarlos siempre integradamente, tanto cuando se diseña como cuando se evalúa una obra lexicográfica.

\section{Sobre el estado de la lexicografía de aprendices}

Reafirmando lo expresado en el párrafo anterior y a la luz de una opinión de Verlinde y Binon (2009: 69) con relación a que "modern pedagogical lexicography mainly developed in Great Britain”, no cabe duda de que es esa tradición lexicográfica la que ofrece el estado del arte en esta materia, a pesar de que en Bugueño Miranda y Reolon

\footnotetext{
${ }^{2}$ A pesar de que Schlaefer (2009: 123), a propósito del concepto de cultura del diccionario [Wörterbuchkultur], sostenga que "im deutschsprachigen Raum Defizite aufweist". La recopilación crítica de Städtler (2003) de 53 proyectos lexicográficos debería ya haber refutado esa afirmación. Además, esta recopilación considera solamente la lexicografía académica [wissenschaftliche Lexikographie]. En el caso alemán, particularmente, hay que agregar también la lexicografía comercial [kommerzielle Lexikographie].

${ }^{3}$ Lexicografía Pedagógica es un término genérico para todos aquellos diccionarios que se emplean tanto para la enseñanza-aprendizaje de la lengua materna como de una lengua extranjera. Corresponde al término inglés Pedagogical Lexicography (cf. HARTMANN; JAMES 2001: s.v.). Bielińska (2010: 18) llama la atención para el hecho de que en la literatura metalexicográfica alemana se usa junto a este término también el término Lernerlexikographie. Para evitar malos entendidos, ya que diccionarios de aprendices [Lernerwörterbücher] y de aprendizaje [Lernwörterbücher] son dos clases de diccionarios que se emplean en la enseñanza de una lengua extranjera, se utiliza en este trabajo el término Lexicografía Pedagógica.
}

Pandaemonium, São Paulo, v. 22, n. 36, jan.-abr. 2019, p. 67-96 
BUGUEÑO MIRANDA, F. - A propósito de três diccionarios de DaF

Jardim (2010) se destaca que los diccionarios de aprendices del alemán presentan una serie de rasgos diferenciales con relación a los del inglés.

Rundell (2008: 221-223) propuso hace casi una década lo que se podría denominar un programa de trabajo tanto para la concepción como para la evaluación de un diccionario de aprendices. Este programa consta de dos máximas (1. la descripción de la lengua en el diccionario debe basarse en evidencia empírica y 2. La presentación de las informaciones debe corresponder a lo que se sabe sobre las necesidades y habilidades lingüísticas del usuario pretendido $)^{4}$. En términos macroestructurales, esto significa que la definición macroestructural cualitativa debe corresponder rigurosamente al vocabulario contemporáneo y frecuente de la lengua [vocabulary control]. Es conveniente recordar que la fundamentación lexicológica de esta premisa corresponde a Nation (2006: 15), quien sostiene que, para el inglés, con el vocabulario de alta frecuencia es posible cubrir el $80 \%$ del vocabulario presente en textos orales y escritos 5 .

En el ámbito microestructural, una cuestión central es el establecimiento y empleo de un vocabulario de definidores [defining vocabulary] para ser empleado masivamente en la generación de paráfrasis explicativas [paráfrases explanatórias] (cf. BUGUEÑo MIRANDA 2009, para una discusión sobre el concepto de paráfrasis explicativa). Igualmente relevantes son las informaciones sobre sintaxis, la presencia de ejemplos y la lematización de unidades fraseológicas.

Tanto Jamada (2015) como Chi (2015) elaboraron una síntesis sobre los avances en la investigación y diseño de diccionarios de aprendices del inglés. Con relación a la definición macroestructural, la preocupación con la lematización de unidades de alta frecuencia constituye, evidentemente, un tópico que no merece discusión. Sin embargo, Chi (2015: 171) destaca que un diccionario de aprendices debe lematizar también "cultural or encyclopaedic aspects of the language". Paradógicamente, afirma también que "High-frequency and function words were discovered to be less demanded". Esto, sin lugar a duda, constituye una innovación en el área. De la misma forma, Chi (2015: 168)

\footnotetext{
${ }^{4}$ Parcialmente, corresponde a Jackson (2002) el mérito innegable de haber elaborado una meritoria síntesis sobre la concepción de un diccionario de aprendices. Cuestiones centrales tales como la necesidad de prestarle atención a las necesidades de producción del aprendiz [encoding needs] - y a las que se les brindará una atención central en este trabajo - parecen no constituir todavía un tópico que preocupe prioritariamente a los especialistas.

${ }^{5}$ No cabe duda, sin embargo, que existe la posibilidad que la misma relación diamésica que existe en inglés no necesariamente exista en alemán, de modo que esta es una variable que requiere una especial ponderación.
}

Pandaemonium, São Paulo, v. 22, n. 36, jan.-abr. 2019, p. 67-96 
BUGUEÑO MIRANDA, F. - A propósito de três diccionarios de DaF

destaca que la progresión alfabética vuelve difícil emplear el diccionario para necesidades de codificación.

En lo que se refiere a aspectos microestructurales, Yamada (2015: 201) defiende el uso de un vocabulario de definidores, ya que "a concept with a complex content is in fact being defined by means of less complex concepts". Sobre este particular, Chi (2015: 173) recuerda que "The traditional defining style uses shorts phrases and synonymous words".

Íntimamente ligado a lo anterior, Chi (2015: 171) destaca la importancia que han ido cobrando los distinguidores semánticos [guidewords], cuya función es ordenar las Lesearten $^{6}$ según núcleos de significación. No obstante, destaca también que los hiperónimos que los constituyen pueden ser ambiguos y confundir al usuario (ibid).

En lo que se refiere a informaciones gramaticales, Yamada (2015: 198) destaca que "The current approach [...] emphasizes what is typical over what is possible". Es necesario resaltar que esto incluye no sólo lo que se refiere a la sintaxis (un factor crucial en el caso del alemán), sino también - y no menos importante en el estado actual de la cuestión - al amplio espectro de las combinatorias léxicas [Wortverbindungen], determinado tanto por su variedad como por la amalgama variable que las caracteriza (cf. WANZECK 2010: 103).

Finalmente, los ejemplos son también un segmento informativo unentbehrlich en un diccionario de aprendices. Chi (2015: 204) destaca que estos pueden ser ad hoc [madeup] o extraídos de corpus [corpus-drived].

\section{Los Lernerwörterbücher del alemán}

Herbst y Klotz (2003: 242) afirman que "Lernerwörterbücher richten sich an Benutzer, die die entsprechende Sprache als Fremdsprache gelernt haben beziehungsweise lernen". Esta definición, esencialmente correcta, requiere, sin embargo, una mayor precisión conceptual. Schlaefer (2009: 188), por ejemplo, destaca que los diccionarios de aprendices "[sc. sind] an den besonderen Anforderungen des Spracherwerbs ausgerichtet", lo que significa, de facto, tres cosas: a) deben estar

\footnotetext{
${ }^{6}$ Leseart "Möglichkeiten der semant. Interpretation eines Ausdruckes" (MLSp 2010: s.v.). Para efectos lexicográficos, es de especial importancia la especificación hecha por WtbLBeg (2002, s.v. Leseart) en el sentido de que una Leseart comporta "[sc. Die] Menge der semantischen Merkmale $+[s c$.die] Menge der Selektionsbeschränkungen eines sprach. Elementes".
}

Pandaemonium, São Paulo, v. 22, n. 36, jan.-abr. 2019, p. 67-96 
BUGUEÑO MIRANDA, F. - A propósito de três diccionarios de DaF

diseñados para un público específico, particularmente en lo que se refiere al nivel de competencia en la lengua extranjera; b) deben poder satisfacer tanto necesidades de recepción como de producción; c) deben ofrecer una imagem linguae no sólo lo más exacta posible, sino que también deben destacar sus particularidades estructurales. Es así como debiera entenderse lo que Schlaefer (2010: ibid) llama de "besondere Anforderungen des Spracherwerbs".

En lo tocante a la lexicografía de aprendices de alemán, resulta preocupante la afirmación de Kispál (2013: 88) al afirmar que "DaF-Wörterbuchbenutzer [sc. verwenden] selten einsprachige Wörterbücher". De facto, Runte (2015: 259), por ejemplo, propone que para tareas de producción el aprendiz emplee un diccionario bilingüe. Así, se vuelve paulatinamente claro que la situación actual de los Lernerwörterbücher está determinada por dos factores intrínsecamente unidos. Por un lado, y a lo que aparece, por una todavía insipiente Wörterbuchkultur con esta clase específica de diccionarios. Por el otro, por el hecho de que están concebidos (todavía) según un perfil de usuario muy genérico.

En términos de inventario Rothenhöfer (2013: 15) enumera un total de 8 diccionarios monolingües y 1 bilingualizado en el ámbito de diccionarios de aprendices de $\mathrm{DaF}^{7}$. Para este trabajo se seleccionaron LGwtbDaF (2015), DuWtbDaF (2015) y HuDuWtbDaF (2007). La selección obedeció al cruzamiento de dos variables. En primer lugar, y en la medida de lo posible, que las obras escogidas atendieran al espectro de niveles de referencia propuestos por el Gemeinsamer Europäischer Referenzrahmen für Sprachen (GER 2001). La aplicación de este parámetro implicó en la elección de las tres obras citadas ad supra: las dos primeras están diseñadas para el estudiante avanzado (C1C2) ${ }^{8}$; la tercera, para el estudiante de ciclos iniciales (A1-B1). En segundo lugar, se procuró también que fueran obras de edición reciente, lo que redundó en escoger LGwtbDaF (2015) y DuWtbDaF (2015). Un caso aparte constituye HuDuWtbDaF (2007). Si bien es cierto que en el intervalo se publicó BaswtbDaF (2013), la desconfianza frente a algunas de las premisas que lo constituirían - tales como la presentación de un nuevo vocabulario básico o el empleo exclusivo de la macroestructura en la generación

\footnotetext{
${ }^{7}$ En BUGUEÑO MIRANDA Y REOLON JARDIM (2010) realizó ya un análisis de cuatro de esos diccionarios: LGwtbDaF (2003), PGwtbDaF (2007), LTwtbDaF (2004) y PBwtbDaF (1999).

${ }^{8}$ De facto, Profile Deutsch (2005, p. 50) alude a LGwtbDaF como la fuente de referencia léxica para los aprendices avanzados.

Pandaemonium, São Paulo, v. 22, n. 36, jan.-abr. 2019, p. 67-96
} 
BUGUEÑO MIRANDA, F. - A propósito de três diccionarios de DaF

de las paráfrasis - llevaron a considerar que HuDuWtbDaF (2007) era una mejor opción para efectos de comparación.

En lo que se refiere a la definición macroestructural, es necesario distinguir entre una definición macroestructural cuantitativa y una definición macroestructural cualitativa (cf. BugueÑo MiRANDA 2007 para una discusión sobre estos conceptos). La definición macroestructural cuantitativa corresponde al número total de lemas que conforman la macroestructura. Hasta ahora la metalexicografía no ha establecido parámetros para su cálculo.

En lo que respecta a LGwtbDaF (2015), a través de un procedimiento que consiste en determinar cuatro intervalos lemáticos de una página cada uno en cuatro puntos diferentes del diccionario y calculando el promedio de lemas de estas cuatro páginas multiplicado por el total de páginas del diccionario se llega a la cantidad de aproximadamente 33.410 lemas. A esa cantidad hay que agregar los 30.000 compuestos y derivados que el diccionario afirma tener (según consta en la contratapa). Si bien no se cuenta con parámetros lexicométricos que permitan hacer proyecciones, es plausible que ese número sea mayor, si se considera la productividad lexicogénica que presentan los Komposita del alemán, que configuran nichos léxicos bajo la forma de run-on-entries. De esta forma, la densidad macroestructural resultante de la adición del número aproximado de lemas más el número de compuestos y derivados suministrado por el propio diccionario bordea los 64.000 lemas 9 .

Ya en el caso de DuWtbDaF (2015) y utilizando el mismo procedimiento anterior, se llega a una densidad mucho menor, con un total aproximado de 14378 lemas. Según consta en la contratapa, el diccionario lematiza 20000 Wörter und Wendungen. Además, se afirma que se optó por una estructura lisa: "Damit vermeidet das Wörterbuch umfangreiche "Nester", die wegen ihrer Komplexität das Auffinden des gesuchten Wortes erschweren" (Front Matter: 11).

HuDuWtbDaF (2007), a su vez, posee una densidad macroestructural cercana a los 15.229 lemas, no obstante que en el Vorwort se señale que "in diesem Wörterbuch finden Sie über 11200 Stichwöter”. La contratapa del diccionario informa, no obstante, que la obra consta con un total de 41500 Stichwörter, Beispiele und Wendungen.

\footnotetext{
${ }^{9}$ Según consta en la contratapa, LGwtbDaF (2015) constaría de "Rund 90.000 Stichwörter, Wendungen und Beispielsätze". Ese cómputo no representa nada en términos metalexicográficos, ya que se mezclan niveles y segmentos informativos de muy diverso orden.

Pandaemonium, São Paulo, v. 22, n. 36, jan.-abr. 2019, p. 67-96
} 
BUGUEÑO MIRANDA, F. - A propósito de três diccionarios de DaF

En lo que respecta a los guarismos que cada diccionario menciona, cabe recordar un desiderátum de Beißwenger y Körkel (2002: 398) con relación a que "von einem Lernerwörterbuch zu erwarten sei [cursiva en el original], daß es insgesamt nur einen recht reduzierten Ausschnitt aus dem Wortschatz der bearbeiten Sprache biete”.

Las cifras ofrecidas por los tres diccionarios merecen tres ponderaciones. En primer lugar, una densidad macroestructural alta no es sinónimo de calidad ni mucho menos de funcionalidad. LGwtbDaF (2015: 7) afirma en el Front Matter que hay un Zentralwortschatz de 5500 lemas que obedece, a lo que parece, al conjunto de intersección de varios vocabularios básicos [Grundwortschätzen] y complementarios [Aufbauwortschätzen] correspondientes al Zertifikatwortschatz del Instituto Goethe. Estas listas se complementaron tanto con las listas de frecuencia del Deutsches Referenzkorpus (DeReKo) del Institut für deutsche Sprache como con las del proyecto Deutscher Wortschatz de la Universidad de Leipzig para establecer las 4000 palabras más frecuentes "soweit sie für Deutschlernenden wichtig erschienen". Möhring y Wallner (2013) hicieron una investigación sobre listas de vocabulario básico fundadas en corpus y cotejadas con el Profile Deutsch y establecieron que hay una falta sensible de coincidencia entre los resultados que se obtienen por criterios estadísticos y los que se obtienen en base al establecimiento de temas, que es el procedimiento que se usa para establecer inventarios léxicos. Este parece ser el modus operandi del Instituto Goethe, a juzgar por páginas como Deutsch am Arbeitsplatz ${ }^{10}$, donde se establece una correlación entre el lugar de trabajo y temas como Berufe y Tätigkeiten am Arbeitsplatz. La red ofrece también la Wortliste del Goethe-Zertifikat $\mathrm{B} 1^{11}$. La exposición de los insumos léxicos exigidos para la competencia léxica de ese nivel sigue también la organización por temas.

Sin duda alguna la densidad macroestructural aproximada que se calculó tanto para DuWtbDaF (2015) como para HuDuWtbDaF (2007) permite suponer que esos números se aproximan más de lo que se podría presumir como una definición macroestructural cuantitativa más acorde con las necesidades del usuario. En este plano cabe sólo trabajar con hipótesis.

Si se considera que ni siquiera hay una fundamentación empírica para establecer cuantitativamente el vocabulario básico del alemán, mucho menos se sabe a cuánto correspondería una macroestructura eficiente, o sea, aquella que coincida con las

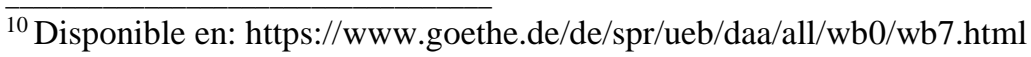

${ }^{11}$ Disponible em: https://www.goethe.de/pro/relaunch/prf/pt/Goethe-Zertifikat_B1_Wortliste.pdf

Pandaemonium, São Paulo, v. 22, n. 36, jan.-abr. 2019, p. 67-96
} 
BUGUEÑO MIRANDA, F. - A propósito de três diccionarios de DaF

necesidades del usuario (cf. BUGUEÑo MIRANDA 2007 para mayores detalles sobre este tema). Considerando la observación de Beißwenger y Körkel (2002) y la falta de estudios lexicométricos es posible afirmar que las aserciones hechas por los diccionarios con relación a la densidad macroestructural no son aussagekräftig. Cabe recordar que la definición macroestructural para esta clase de diccionarios debería resultar del cruzamiento de dos parámetros. Por un lado, aquello que es relevante en términos estadísticos (frecuencia) y, por el otro, en función de los dominios léxicos que el Gemeinsamer europäischer Referenzrahmen für die Sprachen (GER 2001), el Profile Deutsch (2005) y el Zertifikatwortschatz consideren relevantes. La primera dimensión ya ofrece resultados satisfactorios; la segunda todavía no.

En segundo lugar, no es completamente correcto ofrecer guarismos en los que se mezclan dimensiones diferentes de un diccionario. Tanto LGwtbDaF (2015) como HuDuWtbDaF (2007) cuentan los ejemplos como lemas, lo que es completamente improcedente ya que estos constituyen un segmento informativo de la microestructura, mientras que los lemas corresponden a una magnitud macroestructural. En una situación más compleja se encuentra la fraseología (genéricamente denominada de Wendungen). Desde el punto de vista lexicológico, es verdad que el léxico se divide en discurso libre [freie Rede] y discurso repetido [wiederholte Rede], según la ya tradicional distinción propuesta por Coseriu (1992). Como corolario de este principio, hay que aceptar que una unidad fraseológica (como jemandem die Leviten lesen) vale desde el punto de vista de su contenido lo mismo que una unidad unilexemática (como Tür); es decir, es tan palabra como cualquier categoría que constituye el discurso libre. Por causa de su tratamiento de lematización, sin embargo, no es posible disponerlas desplazadas a la izquierda en la progresión lemática vertical. No obstante ello - y dada la completa aleatoriedad con que el discurso repetido se realiza en una lengua - resulta muy difícil comprender cómo se podría contabilizar estas magnitudes léxicas en un diccionario, a no ser, claro está, que se hiciera un recuento de artículo léxico por artículo léxico, lo que parece poco probable.

En tercer lugar - y pensando en usuarios oriundos de tradiciones lexicográficas como la hispánica y la de lengua portuguesa - la opción de estructura lisa es la más apropiada. Resulta sumamente complejo para el usuario leer no sólo en progresión vertical sino también en muchos casos horizontal y calcular, además, los compuestos que corresponden a opciones lexicogénicas a la izquierda o a la derecha del signo lema. Los tres diccionarios emplean, sin embargo, opciones de nicho o nido léxico. LGwtbDaF 
BUGUEÑO MIRANDA, F. - A propósito de três diccionarios de DaF

(2015), por ejemplo, emplea la opción de nicho léxico y nido léxico tanto para los derivados, como s.v. Mittelmaß: “[ ...] mittelmäßig [...], Mittelmäßigkeit”, como también para los compuestos: s.v. Handball: "Handballspiel, Handballspieler, Handballturnier; Feldhandball [...]”. El uso de punto y coma para marcar el cambio de posición no mejora substancialmente la situación. DuWtbDaF (2015) se sirve también - sin mencionarlo explícitamente - de la opción de nido léxico, en la medida en que el lema ocupa la posición derecha del compuesto, interrumpiéndose así la progresión alfabética estricta como s.v. Kessel: “Zus[ammensetzung]. Bergkessel, Gebirgskessel, Talkessel”. De esta forma, no es completamente correcta la afirmación hecha por el propio diccionario y reproducida ad supra que se refiere a que se habría prescindido de Nester. Estos existen aunque, es verdad, no son umfangreich. En HuDuWtbDaF (2007) se emplea también esta opción, como s.v. Matratze: "Zus[ammensetungen]: Federkernmatratze, Schaumgummimatratze". Si bien es cierto que la capacidad del alemán de crear compuestos es notoria, no es menos cierto que las opciones de nido y nicho léxicos dificultan la consulta. LGwtbDaF (2015) es el único diccionario que emplea un realce tipográfico para destacar que el usuario puede buscar también lemas "en sentido horizontal" (dentro del artículo léxico). Los otros dos diccionarios no emplean recurso tipográfico alguno, salvo la abreviatura "Zus". Es altamente criticable que no se asuma explícitamente que se emplean estas opciones de tratamiento lemático; que no se le indique tampoco al usuario que hay casos en los que la búsqueda de unidades léxicas no se ejecuta únicamente en sentido vertical, sino también horizontal, "entrando en el artículo léxico"; por último, que salvo el primero de los diccionarios, no se usen sistemas semióticos diferentes para destacar facta linguae de muy diferente orden.

En el ámbito de la definición macroestructural cualitativa, esto es, qué clase de unidades un diccionario lematiza (cf. BUGUEÑo MIRANDA 2007), lo primero que cabe destacar es que los tres diccionarios reproducen la tendencia de los diccionarios semasiológicos generales del alemán de lematizar afijos. Como ejemplo, se escogieron los prefijos $a b$-, ent-, vor- y $z u$-, presentes tanto en LGwtbDaF (2015) como en DuWtbDaF (2015); HuDuWtbDaF (2007) los lematizó también, con la única excepción de vor-. Ese tipo de elementos lemáticos corresponde a lo que en Bugueño Miranda y Farias (2008) se le denomina "información no discriminante" [informação não discriminante] en la medida en que las instrucciones de uso sobre su significación que se suministran en cada artículo léxico no constituyen una heurística real, pues el usuario no 
BUGUEÑO MIRANDA, F. - A propósito de três diccionarios de DaF

puede crear ad libitum opciones léxicas tales como *abfunktionieren, *entrennen, *vorlernen, o *zugrüßen suponiendo que se trata de sumar simplemente el valor específico de significación de cada prefijo a los verbos respectivos. Las opciones propuestas son opciones de sistema, no de norma (cf. COSERIU 1992 para este concepto). El valor de estos prefijos se establece mucho mejor en cada una sus realizaciones (y de todos los afijos en general) en las paráfrasis explicativas respectivas presentes en los diccionarios.

Una segunda dimensión de la definición macroestructural cualitativa es la distinción e type / token. Esta distinción obedece al principio de establecer (o no) formas preferenciales o canónicas (type) y formas secundarias o no canónicas. Los parámetros para esta distinción pueden afectar a varios niveles de organización de la lengua (ortográficos, fonológicos, morfológicos, léxicos, etc.). LGwtbDaF (2015) es el único de los tres diccionarios que aplicó esta distinción. Esta opción puede realizarse por lematización pareada (Bibliografie, Bibliographie) o por remisión. Una cuestión central en este último caso es que el usuario comprenda el impulso desencadenador de la remisión así como su objetivo (cf. BugUEÑo MIRANDA Y ZANATTA 2010 para un estudio sobre procedimientos medioestructurales). Hay casos clarísimos, tales como los de formas verbales flexionadas, como s.v. dürfte ("Konjunktiv II, 1. und 3. Person Singular $\rightarrow$ dürfen”). En otros casos, la distinción obedece a la variación ortográfica; así acontece s.v. Handicap: [...] $\rightarrow$ Handikap". Incluso, hay casos de variación fonológica: Bẹr-serker, Ber-sẹr-ker. También puede acontecer que se lematice un formante léxico de una unidad fraseológica, como s.v. haushalten: “[...] ID $\rightarrow$ Haus”. Se encontró incluso hasta un caso de typel token por variación diatópica; así s.v. mürb: "besonders süddeutsch A $\rightarrow$ mürbe".

En lo que se refiere a DuWtbDaF (2015), existe solo la opción de lematización pareada, como s.vs. Bibliografie, Bibliographie; Erdgeschoss, Erdgeschoß; Handicap, Handikap; nah, nahe y Ortografie, Ortographie. En estos casos, debe asumirse que el diccionario juzgó que ambas formas son type. Cuando acontece la remisión, como en LGwtbDaF (2015), se le está ofreciendo al usuario una orientación referente a una forma preferencial o, cuando acontece la lematización pareada, que ambas formas son type, como s.v. Bibliografie, Bibliographie.

HuDuWtbDaF (2007) optó también por ofrecer algunos casos de formas variantes, pero ello acontece ya dentro de la microestructura, lo que es un procedimiento muy poco 
BUGUEÑO MIRANDA, F. - A propósito de três diccionarios de DaF

usual (si no único) para el tratamiento de la distinción type / token. Algunos casos son Angler (“[...] Anglerin [...]”); nah (“[...] nahe [...]”); (die) E-mail (“[...] (bes. schweiz.) das E-mail”); Erdgeschoss (“[...] Erdgeschoß [...]”). No es usual que se considere el alomorfo femenino como una forma secundaria. Aún así, esa decisión en términos de posición se haría más afortunada si se hubiera recurrido a la lematización con remisión, es decir, el alomorfo respectivo "debajo" y en bloque independiente del type.

La última dimensión de la definición macroestructural que comporta analizar es la adopción de una solución polisémica u homonímica para el agrupamiento de las paráfrasis, esto es, si independiente o no de consideraciones etimológicas se agrupan o se separan las paráfrasis explicativas en función de criterios tales como la relación etimológica, la afinidad o divergencia semántica, morfológica ${ }^{12}$ y la frecuencia (cf. BUGUEÑo MiRANDA 2016 para una discusión sobre este particular). La tendencia de LGwtbDaF (2015) es adoptar una solución homonímica. Sin embargo, es necesario destacar que no es posible establecer un patrón. Por un lado, está el tratamiento dado a los verbos y, por el otro, a los sustantivos. Con relación a los verbos, se optó por una solución homonímica, como comentado anteriormente, como dürfen ${ }^{1}$, dürfen ${ }^{2}$; folgen ${ }^{1}$, folgen $^{2} ;$ hängen ${ }^{1}$, hängen ${ }^{2}$. No obstante, no es posible establecer con absoluta claridad la razón de la separación de los verbos en artículos independientes ${ }^{13}$. En el caso de dürfen ${ }^{1}$, dürfen ${ }^{2}$, por ejemplo, pareciera que la razón obedece a una diferencia diamésica, ya que para dürfen ${ }^{2}$ se señala que se usa en el registro oral de la lengua (gesprochen). La misma hipótesis se puede plantear para folgen ${ }^{1}$, folgen ${ }^{2}$. Es discutible, sin embargo, si el usuario aprendiz -no necesariamente adentrado en cuestiones de lingüística, además- sería capaz de entender la razón que lleva a estas decisiones. No se debe olvidar jamás que un diccionario comporta una doble heurística: por un lado, de cómo usar la lengua y, por el otro -y no menos importante- de cómo interpretar las propias informaciones que ofrece ${ }^{14}$. En realidad, el segundo es conditio sine qua non para el primero.

El tratamiento de los sustantivos parece guiarse por la dimensión etimológica si se comparan las soluciones propuestas con las que presenta DUW (1999) para las mismas

\footnotetext{
${ }^{12}$ Este aspecto en particular afecta a la lengua inglesa ya que no es necesario ningún expediente formal (derivación) para cambiar de categoría morfológica.

${ }^{13}$ A manera de cotejo y salvo hängen, los otros dos verbos son considerados casos de polisemia por DUW (1999).

${ }^{14}$ Sobre este particular, cabe destacar la afirmación de Thumb (2004: 15) con relación a que "[...] a majority of respondent [sc. of a study] had never received any dictionary use instruction [...]".

Pandaemonium, São Paulo, v. 22, n. 36, jan.-abr. 2019, p. 67-96
} 
BUGUEÑO MIRANDA, F. - A propósito de três diccionarios de DaF

unidades léxicas: Kuli $^{1}$, Kuli $^{2} ;$ Kunde $^{1}$, Kunde $^{2} ;$ Partikel $^{1}$, Partikel $^{2}$; Plastik ${ }^{1}$, Plastik $^{2}$. Al usuario sólo le resta conjeturar si la decisión adoptada obedece al criterio de distinción semántica, que, de facto, se superpone al etimológico, lo que para un aprendiz de DaF es irrelevante, evidentemente.

En lo que toca a DuWtbDaF (2015), se adopta parcialmente el criterio homonímico por diferenciación morfológica en casos como auch ${ }^{1}$ (Adverb), auch ${ }^{2}$ (Partikel); auf $f^{1}$ (Präp. mit Dativ und Akkusativ), auf ${ }^{2}$ (Adverb); erfahren ${ }^{1}$ (Verb), erfahren $^{2}$ (adjektiv); faktisch ${ }^{1}$ (adjektiv), faktisch ${ }^{2}$ (adverb). A ese grupo es necesario agregar también los casos de los sustantivos, tales como Kuli ${ }^{1}$, Kuli $^{2}$; Moment ${ }^{1}$, Moment ${ }^{2}$, $\mathrm{See}^{1}, \mathrm{Se}^{2}$. Las soluciones propuestas se atienen en algunos casos a la etimología; en otros no (como auch ${ }^{1}, a u c h^{2} ; a u f^{1}, a u f^{2}$ ). Sin embargo, para el usuario es una ganancia pues le permite percibir el cambio de categoría morfológica.

HuDuWtbDaF (2007) sigue las mismas decisiones tomadas por DuWtbDaF (2015) sobre este particular. Es decir, la adopción de soluciones homonímicas en auch ${ }^{1}$ (Adverb), auch ${ }^{2}$ (Partikel); auf ${ }^{1}$ (Präp.), auf (Adverb); erfahren ${ }^{1}$ (Verb), erfahren ${ }^{2}$ (adjektiv), See ${ }^{1}, \mathrm{See}^{2}$. Sobre todo en el caso de aprendices iniciales, la solución homonímica es particularmente didáctica por dos razones. Como referido anteriormente, porque permite, en primer lugar, distinguir categorías morfológicas; en segundo lugar, porque la lectura de un artículo léxico les resulta en sí ya difícil, de modo que "dividir" la información en bloques separados contribuye para una schaubare Informationsverdichtung. Este es un aspecto fundamental que la metalexicografia ha relegado a un incomprensible segundo lugar, no obstante el inspirador trabajo de Bray (1989).

Una evaluación parcial de la macroestructura debe hacerse a partir de una doble perspectiva. Por un lado, en función de las estrategias de búsqueda [look-up strategies] (cf. Thumb 2004 para una discusión sobre este concepto). Por el otro, en función de las propias particularidades que presenta el alemán.

Con relación al primer aspecto, es necesario reconocer la completa falta de estudios sobre el particular uso que hacen los aprendices de DaF. La poca evidencia empírica permite afirmar, como referido anteriormente, que la estructura lisa es la más apropiada para este público usuario, en función de que la estructura libre es la estructura de acceso universal. El uso de nichos y nidos léxicos todavía es casi completamente desconocido en muchas tradiciones lexicográficas.

Pandaemonium, São Paulo, v. 22, n. 36, jan.-abr. 2019, p. 67-96 
BUGUEÑO MIRANDA, F. - A propósito de três diccionarios de DaF

Con relación al segundo aspecto, la ausencia de estudios lexicométricos empíricos convierte el establecimiento de una macroestructura eficiente en un desiderátum todavía lejano en la lexicografía de aprendices. Sobrecargar la macroestructura con unidades léxicas de poco valor constituye un exceso que en nada ayuda a un usuario que ya está suficientemente ocupado en interpretar metalingüísticamente una profusa cantidad de informaciones que, a su vez, lo ayudan a resolver tareas en la lengua extranjera. Según Koeppel (2013), LGwtbDaF (2015) es un diccionario para estudiantes avanzados. Si ello es así, cabe preguntarse por qué entre el conjunto de tokens aparecen lematizadas las formas conjugadas de los verbos irregulares, tales como kam, kannte, schrieb y stand, que el aprendiz avanzado debería perfectamente conocer; al mismo tiempo están ausentes, sin embargo, en HuDuWtbDaF (2007), proyectado para un aprendiz elemental que sí necesita ex professo todavía ese tipo de insumo. Lo que sobra en LGwtbDaF (2015) - y ayuda a "hinchar" el diccionario - falta en HuDuWtbDaF (2007), en el que resulta indispensable.

Ya se ha destacado más de una vez ad supra que un diccionario es un instrumento heurístico. Bajo esa premisa, resulta irrelevante también la lematización de afijos. Por un lado, y como comentado anteriormente, el aprendiz sólo podría construir hipótesis léxicas sobre la adición de un afijo a un radical o simplex sin que la forma resultante esté necesariamente realizada en la norma. Por otro lado, sobre todo el aprendiz de ciclos iniciales tiene mucha dificultad para aprender facta linguae tedescae tales como los trennbare y los untrennbare Verben, de modo que es mucho mejor presentarle siempre cada uno de los verbos con sus respectivos afijos. Además, muchos verbos en alemán presentan una modificación de su valencia y de matices aspectuales (cf. DuGram (2009: $\S 1055-158)$ ) a depender de los prefijos respectivos unidos a verbos específicos.

El último aspecto de la definición macroestructural cualitativa o de la adopción de una solución polisémica u homonímica es el que en general los diccionarios presentan los resultados más homogéneos y satisfactorios, sobre todo en lo que respecta a DuWtbDaF (2015) y HuDuWtbDaF (2007), ya que el volumen de informaciones que cada artículo léxico redactado bajo la modalidad de solución homonímica es relativamente reducido. No hay que olvidar que la adopción de una solución homonímica debe siempre pautarse por ofrecerle al usuario un volumen de informaciones que consiga decodificar e interpretar. La única observación crítica que cabe hacer sobre este particular está con relación a que no siempre se consigue comprender bien la razón subyacente que determinó la adopción de esta solución. Ello es fundamental: forma parte de la 
BUGUEÑO MIRANDA, F. - A propósito de três diccionarios de DaF

competencia de consulta de la obra lexicográfica comprender las decisiones adoptadas en la compilación del diccionario. Estas decisiones son instrucciones para el usuario.

En el ámbito microestructural, LGwtbDaF (2015) presenta el programa constante de informaciones (pci) más complejo. En términos de los dos comentarios básicos de la microestructura, comentario de forma y comentario semántico. El primero presenta los siguientes segmentos informativos: representación ortográfica (ins-be-sọ-de-re, ins-besọd-re), separación silábica (lang•sam) y, según la categoría morfológica, flexión de

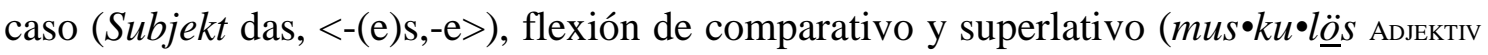
<muskulöser, muskulösest->), posición atributiva o predicativa del adjetivo (per•plex ADJEKTIV meist prädiktiv; gesprochen) y tiempos modelos de la conjugación (schädigen V/T (schädigte, hat geschädigt)), entre otros segmentos informativos. No cabe duda que cada una de estas informaciones constituye lo que en Bugueño Miranda y Farias (2008) se le denomina "informaciones discretas y discriminantes" en la medida en que constituyen hechos de norma (discretos) a los que el usuario les puede sacar provecho heurístico en el uso de la lengua (discriminantes) ${ }^{15}$.

En lo que respecta al segundo, y sin menoscabar las complejidades estructurales que caracterizan la morfología del alemán, es este siempre un desafío tanto para el compilador del diccionario como para el propio usuario.

Para efectos de análisis, es recomendable distinguir entre segmentos informativos que atienden a las tareas de recepción y aquellos que atienden a tareas de producción textual.

En este sentido, no cabe duda de que la paráfrasis explicativa ocupa el primer lugar entre los segmentos para la recepción. Es fundamental dejar muy en claro que la redacción de paráfrasis siempre presentará resultados muy heterogéneos en cualquier diccionario por la simple razón de que hay palabras difíciles de definir (cf. BUGUEÑo MIRANDA Y FARIAS 2013 para una discusión sobre este tópico). El uso de un vocabulario

\footnotetext{
${ }^{15}$ En el caso de diccionarios de aprendices, esta doble condición o el caso de que toda información del diccionario debe corresponder rigurosamente a un hecho de norma (información discreta) que le permite al usuario sacar provecho en el uso de la lengua (información discriminante) - parece casi una obviedad, pues se espera que un diccionario se atenga rigurosamente a este principio. Sin embargo, en diccionarios de aprendices del inglés hay casos en los que la presentación de las informaciones, no obstante correspondan a hechos de norma, no son necesariamente discriminantes, pues un lay-out deficiente no permite que el usuario comprenda la naturaleza del hecho lingüístico descrito lexicográficamente.
} 
BUGUEÑO MIRANDA, F. - A propósito de três diccionarios de DaF

controlado de definidores [kontrollierter Definitionswortschatz ${ }^{16}$ ayuda, evidentemente, pero, como resaltan HeRBST y KLOTZ (2003: 48) "es dürfte kaum möglich sein, alle Bedeutungen [...] im Rahmen [sc. eines begrenzten Definitionswortschatzes] semantisch befriedigend zu erklären [...]”. En general, las paráfrasis en LGwtbDaF (2015) se demuestran bien estructuradas y de fácil comprensión, como, por ejemplo, s.v. Fahrstuhl "eine Kabine, mit der Personen in einem Gebäude nach oben und unten transportiert werden" $" 17$. Evidentemente, cuando se trata de sustantivos o adjetivos abstractos, los resultados suelen ser menos logrados; así, por ejemplo, s.v. eitel "<ein Mensch>so, dass er bewundert werden will und sich daher in besonderer Weise benimmt oder kleidet”. El tratamiento de los verbos amerita, sin lugar a dudas, un comentario por separado, ya que la generación de la paráfrasis explicativa debe considerar las especificidades de valencia que cada verbo per se presenta. Un ejemplo paradigmático es el caso del verbo geben:

ge•ben $[\ldots]$ — V/T $\$ einer Person;Sache,Geld 1 jemandem etwas geben etwas in die Hände oder in Nähe einer Person legen oder stellen, sodass sie es bekommt $\leftrightarrow$ nehmen |jemandem ein Buch geben | einem Kind ein Glas Milch geben [...] 9 (jemandem) etwas geben verwendet zusammen mit einem Substantiv, um ein Verb zu umschreiben | (jemandem) eine Antwort geben jemandem antworten | (jemandem) einen Befehl geben (jemandem) etwas befehlen [...] - einer Person; am Telefon 10 einer Person jemanden/etwas geben jemanden am Telefon mit der genannten Person oder einem Mitarbeiter der genannten Abteilung o Ä. sprechen lassen |Geben Sie mir bitte die Versandabteilung | Ich gebe Ihnen Herr Müller [...]. LGWTBDAF (2015: s.v.)

Lo primero que cabe destacar es el uso de distinguidores semánticos ${ }^{18}$ [Wegweiser ${ }^{19}$ ] en un artículo léxico particularmente extenso ( 2 columnas). Estos distinguidores semánticos cumplen un doble objetivo. Además de agrupar Lesearten (ver nota n. 5), establecen cómo llenar los actantes; en este caso, el dativo (einer Person) y el acusativo (Sache, Geld). Una de las características de la lexicografía del alemán es la recursividad de los segmentos informativos, es decir, su repetición. En este caso, el distinguidor semántico se vuelve a repetir una segunda vez en la fórmula jemandem etwas

\footnotetext{
16 Engelberg y Lemnitzer (2004: 231) lo definen como "[...] der Wortschatz, der in den Bedeutungserklärungen verwendet wird. Besonders Lernerwörterbücher bemühen sich, den Unfang des Definitionswortschatzes zu kontrollieren, d.h. auf eine festgelegte Menge von lexikalischen Einheiten zu beschränken".

${ }^{17}$ Otros ejemplos de definiciones bien estructuradas y de fácil comprensión son dampfen, Handfertigkeit, Konditor y niedlich, entre muchos casos.

${ }^{18}$ En inglés, semantic markers. Un distinguidor semántico es un mecanismo heurístico para reunir grupos de significación análogos (Lesearten) o, como en este caso, de comportamientos actanciales comunes a varias Lesearten. Es un mecanismo heurístico pues su objetivo es permitirle al usuario un Überblick sobre la multiplicidad de significación que una unidad léxica posea, así como facilitarle la búsqueda dentro de artículos léxicos largos y/o altamente polisémicos.

${ }^{19}$ LGwtbDaF (2015: 29) propone el término Wegweiser.
}

Pandaemonium, São Paulo, v. 22, n. 36, jan.-abr. 2019, p. 67-96 
BUGUEÑO MIRANDA, F. - A propósito de três diccionarios de DaF

geben y, por último, en los ejemplos (jemandem ein Buch geben, einem Kind ein Glas Milch geben). Esta recursividad es fundamental como un mecanismo complementario cuando la paráfrasis explicativa no consigue ser completamente clara, lo que acontece, por ejemplo, en la acepción 10. El distinguidor semántico marca el dativo; am Telefon es un frame que el usuario solo consigue interpretar cabalmente por el ejemplo. La paráfrasis no es completamente clara, ya que es difícil establecer cómo llenar los actantes.

Otro segmento que se adscribe a la función de recepción es la sinonimia. En general, esto acontece con un doble propósito. El sinónimo es otra designación para el mismo contenido sin que se explicite el contenido común de ambas expresiones (tertium non datur); al mismo tiempo constituye una remisión para otro artículo léxico. Ejemplos: Glace $[\ldots]]_{\mathrm{cH}} \approx$ Speiseeis, Eiscreme; Leihaus $[\ldots] \approx$ Pfandhaus. Sobre el valor del signo $\approx$, ver ad infra las observaciones de la sinonimia con fines de producción.

Un último aspecto de segmentos informativos para la función de recepción es el empleo de sustitución ostensiva, o sea, el uso de figuras que aparecen como representamen de una unidad léxica (cf. BUGUEÑo MIRANDA 2009 para una discusión sobre este tópico). Ejemplos de sustitución ostensiva acontecen s.vs. Brezel, Deckel, Feder, lang, leer, Pfeife, Spitze. La sustitución ostensiva es particularmente útil cuando, por efecto del principio del anisomorfismo lingüístico (cf. ZGUSTA 1971 para este concepto), un número de entidades léxicas recibe una misma designación, mientras que lo mismo no acontece necesariamente en otra lengua. Así, por ejemplo s.v. Deckel se representan los artefactos que en alemán se designan por Deckel y aquellos que, cumpliendo la misma función, se designan por Kappe. En el caso de Feder, por ejemplo, el alemán usa la misma designación tanto para las formaciones córneas de que está cubierto el cuerpo de las aves como para los resortes. Es necesario reconocer, sin embargo, que la investigación sobre los mecanismos de sustitución ostensiva ofrece todavía resultados muy modestos.

Ad supra se señaló que era apropiado separar entre los segmentos informativos destinados a la recepción y aquellos concebidos para la producción. Cabe señalar que la explicitación de la valencia cumple una función doble. Sirve para la interpretación apropiada de un enunciado, pero al mismo tiempo es un Muster de cómo estructurar sintácticamente un enunciado, cumpliendo así también con la función de producción. Por ejemplo, s.v. verändern, aparecen las siguientes especificaciones: “[...] V/T 1 jemanden/etwas verändern bewirken, dass jemand/etwas anders wird [...] | Das Kind 
BUGUEÑO MIRANDA, F. - A propósito de três diccionarios de DaF

hat unser Leben sehr verändert [...]”. En este caso, para efectos de producción, se le informa al usuario que el objeto directo de verändern puede ser de persona (jemanden) o de cosa (etwas). Nótese que la entidad de persona o de cosa vuelve a aparecer en la paráfrasis, pero cumple esta vez la función de sujeto en la oración subordinada. Estos cambios de función, pero no de identidad ontológica, suelen ser de difícil comprensión para los aprendices, de modo que el ejemplo cumple también una doble función, no sólo para elevar la comprensión de la paráfrasis, sino también para ayudar a calcular la explicitación de la estructura sintáctica que el verbo comporta.

Otro segmento informativo que cumple una doble función es la sinonimia. Los casos de equivalencia van precedidos del marcador $\approx$, que cumple dos tareas: por un lado, es el indicador estructural de sinonimia propiamente tal; por el otro, indica que la equivalencia es aproximada (como s.v. Marihuana) o parcial (la sinonimia existe sólo con relación a una determinada Lesert, como s.v. organisieren y Studio). Ejemplos: Marihuana $[\ldots] \approx$ Haschisch; organisieren $[\ldots] 2[\ldots] \approx$ stehlen; Studio $[\ldots] 2[\ldots] \approx$ Atelier.

Un tercer segmento informativo para fines de producción es la antonimia. LGwtbDaF (2015) relaciona cada opción antonímica ofrecida a una determinada Leseart dentro del artículo léxico, lo que se constituye una decisión acertada si se piensa que las relaciones antonímicas son tanto o más complejas que las sinonímicas (complementarias, contradictorias, conversas, etc.; cf. WANZKEK 2010). Así, por ejemplo, s.v. heben "[...] 3 etwas hebt etwas [...] etwas verbessert, steigert etwas [...] $\leftrightarrow$ senken [...] 5 etwas hebt sich etwas wird nach oben bewegt, geht in die Höhe $\leftrightarrow$ senken [...]"; s.v. mager “[...] 1 (von Tieren und Menschen) mit wenig Muskeln und wenig Fett $\leftrightarrow$ dick $[\ldots] 2$ mit wenig oder gar keinem Fett <Fleisch, Schinken, Käse> $\leftrightarrow$ fett [...]”; s.v. Profi “[...] eine Person, die besonders eine Sportart beruflich ausübt $\leftrightarrow$ Amateur [...]”.

Sin lugar a duda, las colocaciones y las coligaciones corresponden a aquellos segmentos informativos fundamentales para la producción textual. LGwtbDaF (2015) se demuestra un diccionario extremadamente abundante tanto en patrones colocacionales como coligacionales. Los patrones colocacionales son presentados dentro de paréntesis triangulares y relacionados también a una Leseart específica, como, por ejemplo, s.v. empfangen

[...] $1[\ldots]$ etwas (von jemandem) bekommen <ein Geschenk, ein Telegramm, einen Brief, einen Auftrag empfangen> 2 [...] jemanden (irgendwie) begrüßen <jemanden freundlich, höflich, herzlich, kühl empfangen> [...]. LGWTBDAF (2015) 
BUGUEÑO MIRANDA, F. - A propósito de três diccionarios de DaF

Otro ejemplo se encuentra s.v. kongruent " $[\ldots]<$ Begriffe, Figuren, Flächen Zahlen> [...]".

En lo que respecta a patrones coligacionales, se ofrecen profusamente informaciones sobre este particular, como s.v. beinahe "[...] beinahe und fast können ein Verb modifizieren, nahezu jedoch nicht [...]"; s.v. dürfen ${ }^{2}$

[...] 1 [...] als Objekt steht meist es, das, dies oder viel, wenig, einiges, nichts [...] 2 [...] Als Vollverb ist die Form im Perfekt gedurft; als Modalverb zusammen mit einem Infinitiv ist es dürfen: Das hätten wir früher nicht gedurft / nicht tun dürfen.

También en los llamados Infokasten, que son formas de Middle-Matter, se encuentran informaciones sobre patrones coligacionales, como s.v. damit.

La larga exposición que ha significado el análisis de la microestructura de LGwtbDaF (2015) demuestra que el diccionario posee una microestructura densa e intensa (cf. BUGUEÑO MIRANDA 2009 sobre este particular), esto es, formada por una gran cantidad de segmentos informativos, muchos de los cuales son recursivos.

Desde la perspectiva del usuario, la recursividad constituye un mecanismo de feedback al que se le puede sacar un provecho indudable, sobre todo cuando cumple con la tarea de complementar otros segmentos, tales como las paráfrasis y la explicitación de la valencia. No obstante es necesario reconocer que puede constituir también una sobrecarga en la medida en que el volumen de informaciones que el usuario debe procesar es considerablemente alto.

Es necesario destacar también que la constante presentación de patrones combinatorios es un mérito intrínseco de LGwtbDaF (2015), porque atiende a una triple demanda. En primer lugar, a una orientación específica del GER (2001 §, 5.2.1.1 a, feste Kollokationen) en la medida en que la dimensión sintagmática es un aspecto central de la competencia léxica; en segundo lugar, a lo que la teoría lingüística ha logrado descubrir cómo se comportan las lenguas naturales (the idiom principle) y, por último, al ofrecerle al aprendiz insumos esenciales para incrementar su competencia lingüística.

Con relación al segundo diccionario en análisis, DuWtbDaF (2015), lo primero que se puede apreciar es que los artículos léxicos son claramente más sucintos en comparación con LGwtbDaF (2015). El pci es básicamente el mismo, aunque no existe la recursividad que se destaca en el caso anterior. Como segmentos estándares se pueden mencionar la separación silábica (he $|r|$ ein, Roll $\mid$ Trep $\mid p e$ ), transcripción fonética (s.v. klappern: ['klapən]; s.v. Maßnahme: ['m:sna:mə]), indicación de género a través del 
BUGUEÑO MIRANDA, F. - A propósito de três diccionarios de DaF

artículo (s.v. Becher [...], der; s.v. Unwetter [...] das) y, como es práctica en la lexicografía alemana, los morfemas de caso: genitivo y de nominativo plural (s.v. Bild [...]-[e]s, -er). En este contexto es interesante destacar los casos en los que se presentan formas que se consideran excepciones al paradigma de formación de plural, como s.v. Büffet ([...]-[e]s, -s und-e, auch: Buffet [...]-s, -s), s.v. Parkplatz ([...]-es, Parkplätze), así como casos de pluralia tantum, como s.v. Flitterwochen ([...] <Plural>).

De forma análoga al diccionario anterior, conviene distinguir, en el ámbito del comentario semántico, entre segmentos destinados a la recepción y a la producción. Las paráfrasis obedecen, evidentemente, a la función de recepción. Lo mismo que vale para LGwtbDaF (2015) vale también para DuWtbDaF (2015), así que se nota que hay algunas paráfrasis bien estructuradas y otras que presentan déficit.

Ejemplos de paráfrasis bien estructuradas se encuentran s.v. belanglos "ohne große Bedeutung, nicht weiter wichtig”, s.v. leserlich "gut zu lesen, zu entziffern”, s.v. Molkerei "Betrieb, in dem Milch verarbeitet wird" o s.v. zuvorkommen "schneller sein (als eine andere Person, die das gleiche tun wollte)". Estas paráfrasis pueden ser consideradas como bien estructuradas ya que emplean un vocabulario y una sintaxis simples; además, las unidades léxicas definidoras forman parte del inventario léxico de los niveles de referencia $\mathrm{A} 1$ a $\mathrm{B} 1^{20}$.

Otras paráfrasis no se demuestran de fácil inteligibilidad, como, por ejemplo s.v. flott" 1 . in einem angenehmen, erfreulichen Tempo [von sich gehend, erfolgend], schnell, flink: eine flotte Bedienung [...] 2. a) schick, lustig-locker [aussehend]: ein flotter Hut b) (von Personen) hübsch, attraktiv [und unbekümmert]: ein flottes Mädchen [...]”. En este caso concreto, es la suma de los distinguidores semánticos (como aussehend o hübsch) y el ejemplo lo que ayuda a tornarla comprensible (cf. FARIAS 2008 para una discusión sobre el valor del ejemplo). Otro ejemplo es la paráfrasis ofrecida s.v. unwillig "Unwillen empfindend, erkennen lassend: er schüttelte unwilling den Kopf, sehr unwillig putzte sie jeden Freitag die Treppe". En este caso, hay tres factores que comprometen su comprensión. En primer lugar, está el empleo del sustantivo abstracto Unwillen (pl.), lo que le confiere a la paráfrasis un sesgo de inevitable circularidad. Evidentemente, le cabe al usuario la opción de consultar s.v. Unwille ("Missfallen, das sich in Ungehaltenheit, Gereizheit, unfreundlicher oder ablehnender Haltung äußert”), aunque esta operación no

\footnotetext{
${ }^{20}$ Empleándose como referencia los inventarios léxicos de A1 a B1 que constan en HuDuWtbDaF (2007), la única unidad que no aparece en esos inventarios es verarbeiten.

Pandaemonium, São Paulo, v. 22, n. 36, jan.-abr. 2019, p. 67-96
} 
BUGUEÑO MIRANDA, F. - A propósito de três diccionarios de DaF

necesariamente traerá una claridad sobre el significado de unwillig. Un segundo factor es el uso del gerundio. Las construcciones con esta forma no personal del verbo conllevan un matiz aspectual durativo de compleja comprensión también.

Finalmente, los ejemplos tampoco contribuyen significativamente como indicios que le permitan al usuario deducir el significado. Otro ejemplo en este sentido es la paráfrasis ofrecida s.v. Kasten "rechtwinkliger, aus festem Material bestehender Behälter (meist mit Deckel), der für die Aufbewahrung, den Transport o. Ä. unterschiedlicher Dinge bestimmt ist". En este caso concreto, la opción de la sustitución ostensiva habría contribuido enormemente para permitir una comprensión del significado de Kasten.

Ad supra, ya se ha insistido que este es el cuadro general que presentan las paráfrasis no sólo de diccionarios de aprendices, sino también de diccionarios semasiológicos en general. Por ello, un análisis de este aspecto no podría terminar sin resaltar los esfuerzos de los redactores de DuWtbDaF (2015) por elucidar el significado de muchas palabras. Para ello, el diccionario dispone de cuadros en los que se explican las diferencias de significado, como por ejemplo, elektrisch / elektronisch, Kosten / Unkosten, meiden / vermeiden, naturgemäß /natürlich. Las extensas explicaciones - y no paráfrasis -, así como el constante contraste con el otro par del binomio permiten que el usuario consiga establecer perfectamente la diferencia entre las dos palabras.

Dadas las particularidades que los verbos ofrecen, esta categoría merece una evaluación por separado. La valencia se ofrece en algunos casos de una manera muy simple, como s.v. kleben "1. (tr.: hat) mithilfe von Klebstoff an/auf/in etwas befestigen: eine Briefmarke auf die Postkarte, Fotos ins Album kleben”. En otros casos, la conjunción de la paráfrasis y los indicadores estructurales no son suficientes para establecer el patrón sintáctico, como s.v. zeigen "1. < itr.; hat> mit dem Finger, Arm eine bestimmte Richtung angeben, ihn auf jmdn., etwas, auf die Stelle, an der sich jemand befindet, richten und damit darauf aufmerksam machen: sie zeigte [mit dem Finger] auf das Haus [...]”. En tal situación, es el ejemplo el que permite comprender las particularidades sintácticas del verbo.

No es posible concluir el análisis de los verbos sin referirse también a casos en los que la descripción resulta excesivamente compleja para un aprendiz. Así, por ejemplo, s.v. vorschlagen se ofrece la siguiente descripción sintáctica: "<tr.; hat $>$ a) als mögliche und empfehlenswerte Vorgehensweise, Handlungsweise nennen, anbieten: ich schlage vor, wir gehen jetzt nach Hause; er schlug ihr vor, mit ihm nach kommen; ich schalge

Pandaemonium, São Paulo, v. 22, n. 36, jan.-abr. 2019, p. 67-96 
BUGUEÑO MIRANDA, F. - A propósito de três diccionarios de DaF

ihnen dieses Hotel vor [...]". El indicador estructural "tr." señala claramente que el verbo se construye con objeto directo. La paráfrasis, no obstante, presenta dos graves problemas. En primer lugar, la proposición que la constituye ofrece las expresiones complementares (y disyuntivas) Vorgehensweise, Handlungsweise y nennen, anbieten, de modo que le cabe al usuario escoger, según las restricciones contextuales ${ }^{21}$ en que se encuentre empleado el verbo vorschlagen. Los redactores de diccionarios emplean estos mecanismos para reducir la vaguedad (vagueness) que caracteriza a tantas unidades de la lengua cuando están contextualmente insertas (cf. KILGARRIFF 2008 para una discusión sobre el rol de este fenómeno en la descripción lexicográfica). De esta manera, las formas alternativas permiten "acomodar" mejor la operación de sustitución del definiens al tratar de establecer una Leseart. Ayudaría a una mayor comprensión del papel de estas unidades si se emplearan barras diagonales (/). En segundo lugar, la proposición que aparece como definiens, emplea también un verbo transitivo (nennen, anbieten). Sin embargo, no hay ningún indicador estructural (como etwas, por ejemplo) que ayude al usuario a completar el contenido proposicional. En tercer lugar, al evaluarse los ejemplos, resulta perceptible que el primero de ellos usó lo que DuGram $(2009$, §1342) denomina uneingeleiteter Nebensatz, o sea, una oración subordinada con el nexo subordinador dass elidido. Esta opción es plenamente lícita en la lengua, pero hay que cuestionarse si el didactismo no debería prevalecer, esto es, si no habría que construir el ejemplo con la oración subordinada introducida por dass. Finalmente, no es posible dejar de mencionar que la descripción sintáctica tampoco destaca la opción de dativo con este verbo. Dos de los ejemplos realizan este actante.

En lo tocante a segmentos para la producción, la descripción sintáctica de los verbos, evidentemente, sirve también para esos fines. Otro segmento para la producción, y que DuWtbDaF (2015) marca explícitamente, es la sinonimia. Lo interesante es que las opciones sinonímicas aparecen relacionadas directamente a cada Leseart dentro del artículo léxico. Así, por ejemplo, s.v. herunterkommen: “a) in einem gesundheitlich, moralisch, wirtschaftlich schlechten Zustand befindlich [...]. Syn.: bankrott, pleite, ruiniert [...]; b) in äußerlich schlechten Zustand [...]. Syn.: verfallen, verkommen”. Hay también presentación de patrones colocacionales, pero no aparecen destacados como un segmento per se, sino que forman parte del segmento de los ejemplos. S.v. Beere "[...]:

\footnotetext{
${ }^{21}$ Es decir, al conjunto de expresiones que preceden y siguen a una determinada unidad lingüística. Cf. DLexFr (2017, s.v. contexte).

Pandaemonium, São Paulo, v. 22, n. 36, jan.-abr. 2019, p. 67-96
} 
BUGUEÑO MIRANDA, F. - A propósito de três diccionarios de DaF

Beere pflücken, sammeln”; s.v. deuten: “[...] 2. [...] Träume, Zeichen deuten [...]”; s.v. Gespräch: “[...] ein Gespräch führen, an einem Gespräch teilnehmen [...]”. En estas condiciones, su utilidad es escasa, ya que difícilmente el usuario interpretará estas informaciones como una clase de combinatorias.

Es evidente que la microestructura de DuWtbDaF (2015) puede ser calificada como una microestructura simple, constituida por un programa constante de informaciones reducido a los segmentos básicos que se esperan en un diccionario de aprendices. Esa simplicidad se traduce en informaciones que no presentan especial dificultad para un aprendiz. Los aspectos críticos que el análisis revela obedecen, sin embargo, a problemas que rebasan la concepción de este o de cualquier diccionario de orientación semasiológica y que son aquellos relacionados a la generación de paráfrasis. En este sentido, sin embargo, un uso de mecanismos complementarios, tales como las figuras, constituye una ayuda a una mejor comprensión de las paráfrasis. Ad supra, se comentó ya que todavía se sabe poco sobre este tema. Sin embargo, si se asume que toda paráfrasis tiene una dimensión intencional y otra extensional, una paráfrasis deficitaria no permite que se reconozca la entidad extralingüística denotada por la proposición. Es ya en ese momento en que debe pensarse en la opción de sustitución ostensiva. En lo tocante a la valencia, es extraño que DuWtbDaF (2015) no le preste la debida atención, considerando que se trata de un rasgo estructural particularmente complejo para un aprendiz. Lo mismo vale para las colocaciones.

El análisis del pci de HuDuWtbDaF (2007) seguirá, evidentemente, el patrón empleado hasta aquí, o sea, comenzando con una evaluación de los segmentos del comentario de forma. Este está conformado por la indicación ortográfica, silábica, de género, de número y la representación fónica.

Al lema, evidentemente, están integradas la indicación ortográfica y la división silábica, como s.v. spä|ter; Un|ge|zie $\mid$ fer y Zu|hau|se. Se detectó también la presencia de representación ortográfica y división silábica alternativa (type / token) ${ }^{22}$, como s.v. Ma $\mid$ yon $\mid$ nai $\mid$ se [...] Ma |jo | nä| se. Un Aspecto especialmente sorprendente es la marcación del género por medio de la anteposición del artículo respectivo al lema: das Hin $\mid$ der $\mid$ nis; die Ent $\mid$ ste $\mid$ hung $\mid$; der Lack. Para estudiantes de nivel elemental esta decisión es una ayuda indudable, si se considera que el aprendiz de alemán no sólo debe

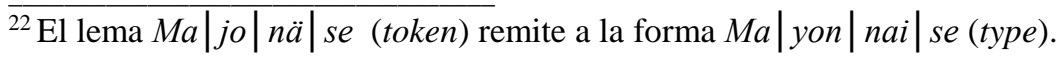

Pandaemonium, São Paulo, v. 22, n. 36, jan.-abr. 2019, p. 67-96 
BUGUEÑO MIRANDA, F. - A propósito de três diccionarios de DaF

apropiarse de una lengua que per se ya es compleja, sino que además debe familiarizarse con el metalenguaje que todo diccionario de aprendices supone. En este mismo ámbito, cabe señalar también la presencia de casos de lematización por alomorfia de género, como s.v. Erbin “[...] weibliche Form zu $\uparrow^{2}$ Erbe". El tratamiento de este fenómeno se da por lematización separada (como el caso de Erbin) o por lemartización pareada, como s.v. $\operatorname{der}$ Arzt [...], die Ärz| tin; der Fri| seur [...], die Fri $\mid$ seu $\mid$ rin; der In $\mid$ ge $\mid$ ni $\mid$ eur [...] die In $|g e| n i|e u| \operatorname{rin}[\ldots]$, etc. No es posible determinar, sin embargo, qué principio subyace para que en un caso se haya optado por la lematización separada y en los otros por lematización pareada. Toda disposición de la información en un diccionario debe obedecer a un principio táctico, esto es, debe suponer algún rasgo relevante con relación a la lengua descrita. De la misma forma, se prestó también atención a la marcación de género cuando ello conlleva distinciones diatópicas, tales como s.v. der Mar|zi|pan “[...]; (österr.:) der; [...]".

En lo tocante a la indicación de número, esta sigue también un patrón ya tradicional en diccionarios de alemán, ofreciéndose siempre las diferentes modalidades de su realización, como s.v. der Gast $\mid$ ar $\mid$ bei $\mid$ ter “[...] -“; der Gast $\mid$ ge $\mid$ ber [...], die Gast $\mid$ ge $\mid$ be $\mid \operatorname{rin}[. .$.$] - nen [...]; der Gast \mid$ hof "[...] Gasthöffe [...]"; die Gast $\mid$ stät $\mid$ te "[...] -n [...]"; der Fach $\mid$ mann "[...] Fachleute [...]". Un hecho digno de notarse es la explicitación de ciertos patrones coligacionales con relación al uso del artículo en plural, como s.v. die $I l \mid$ lus $\mid$ trier $\mid$ te “[...] (ohne »die« in Plural: [viele] Illustrierte) [...]".

El último aspecto del comentario de forma que corresponde tratar es la transcripción fonética. La representación de este segmento informativo sigue los patrones ya consagrados sobre el particular, o sea, se trata de una transcripción estrecha, como s. v. die Bu|che "[’bu:xə] [...]"; s.v. fürch|ter|lich "['fYrçtelirç]; s.v. das Miss |ver|ständ| nis "['misfevftentnis] [...]". Naturalmente, se podría cuestionar en qué medida un aprendiz de nivel inicial, y que probablemente carece de conocimientos metalingüísticos, tendría la capacidad de representarse el respaldo fónico de cada transcripción. Si los diccionarios de aprendices estuvieran diseñados para públicos con un background lingüístico específico, se podría emplear un sistema de transcripción que usara las letras del alfabeto de la lengua materna de los aprendices en aquellos casos, evidentemente, en que hubiera una convergencia fónica entre una letra del alemán y la respectiva de su lengua materna. Con el advenimiento del soporte digital, la lexicografía inglesa, particularmente, ofrece junto con el diccionario impreso acceso a una fuente 
digital que permite escuchar la pronunciación de cada lema. Cabe todavía investigar, sin embargo, si tal opción le es realmente útil al usuario de ciclo inicial, considerando que está todavía en una fase de desarrollo de la competencia fonológica.

Siguiendo la estructura general de análisis, toca ahora referirse a las paráfrasis, que corresponden a un segmento informativo para fines de recepción, como se ha establecido ya. En este aspecto en particular, se percibe claramente que fueron redactadas pensando en un usuario con conocimientos insipientes de la lengua. En términos generales, aparecen bien estructuradas. Sirvan como ejemplos los casos s.v. die Fahn $\mid$ dung "[...] polizeiliche Suche [...]"; s.v. der He|li|kop $\mid$ ter (hay una remisión a Hubschrauber: "eine Art Flugzeug mit Flügeln, die sich waagerecht drehen"; no obstante la paráfrasis no ser muy clara, ya que prototípicamente son los aviones los que tienen Flügeln (los helicópteros poseen Rotorblätter, una palabra demasiado difícil para estudiantes de ciclo inicial), ese déficit se compensa muy bien con una sustitución ostensiva, que corresponde, evidentemente, al dibujo de un helicóptero; s.v. krab|beln “[...] 1. (von Kleinkindern) sich auf Händen und Knien fortbewegen [...]. 2. (von kleinen Tieren) laufen [...]" (en este caso, los distinguidores semánticos entre paréntesis ayudan a determinar la Leseart respectiva); s.v. die Lü|ge "[...] falsche Aussage, die bewusst gemacht wird und jmdn. täuschen soll [...]"; s.v. nahr| haft "[...] reich am Stoffen, die für den Körper wichtig sind [...]”; s.v. der ${ }^{1}$ Schein “[...] 1. Licht [...] 2. [falscher] äußerer Eindruck [...]"; wahr [...] der Wahrheit, der Wirklichkeit, den Tatsachen entsprechend [...]". En general, las paráfrasis están bien estructuradas. Se usa un vocabulario relativamente simple (al costo de algo de pérdida en el plano intencional, como s.v. Hubschrauber, sin embargo). La extensión de las paráfrasis se caracteriza por enunciados breves. Evidentemente, como en el caso de la definición de los adjetivos, no siempre es posible generar una paráfrasis que en la que el isomorfismo morfológico se manifiesta por un adjetivo seguido de determinantes, como es el caso de nahrhaft. En otros casos, como s.v. wahr, y siguiendo un patrón sintáctico común del alemán, se empleó el expediente de la inversión ("der Wahrheit ...entsprechend"), lo que eventualmente le puede causar dificultades de comprensión a un estudiante de ciclo inicial.

La descripción sintáctica de los verbos no escapa al patrón generalmente adoptado no sólo por los diccionarios de aprendices, sino también por los diccionarios semasiológicos generales del alemán. S.v. ein $\mid$ ge $\mid$ wöh $\mid$ nen “[...] <sich [irgendwo] e.> [...]: hast du dich in der neuen Schule schon eingewöhnt?; sie hat sich inzwischen bei uns 
BUGUEÑO MIRANDA, F. - A propósito de três diccionarios de DaF

eingewöhnt [...]"; s.v. för $\mid$ dern "[...]<tr.; hat; jmdn., etw f.>; s.v. sa $\mid$ gen “[...] 1. <etw. s.> sprechen, äußern [...] 2. < [jmdm. etw. s.> [jmdm.] etwas mündlich mitteilen [...] 3 <etw. s.> behaupten [...]". La descripción es clara y discriminante, ya que le ofrece al estudiante un conjunto variado de informaciones altamente útiles. Así, por ejemplo, s.v. eingewöhnen, y entre corchetes, se indica que el verbo tiende a usarse con un locativo. Los dos ejemplos presentados realizan esta opción. En lo tocante a fördern, por otra parte, los pronombres jmdn. y etw. indican que el objeto directo puede ser de cosa o de persona. Finalmente, en el caso de sagen, el cálculo de valencia está íntimamente ligado a cada Leseart, cuestión que aparece claramente discriminada.

En el ámbito de la producción, HuDuWtbDaF (2007) ofrece dos segmentos explícitos y uno implícito. Son segmentos explícitos la sinonimia y la antonimia. Con relación a la primera, cabe señalar que se adoptó acertadamente la decisión de relacionar las opciones sinonímicas a cada Lesart dentro de un artículo polisémico, como s.v. häss $\mid$ lich “[...] 1. nicht schön [...]. Syn.: grässlich (ugs.), scheußlich. 2. gemein [...]. Syn.: eklig, fies (ugs.); s.v. Mi|ne "[...] 3 Bergwerk unter der Erde [...]. Syn.: Grube. Con relación a la antonimia, se pueden citar casos de este segmento s.v. klein "[...] / Ggs., groß/ [...]"; s.v. oben "[...] /Ggs. unten/ [...]"; s.v. un $\mid$ be $\mid$ weg|lich “[...] 2. nicht beweglich /Ggs. beweglich/ [...]".

En una situación incomprensiblemente extraña se encuentra el tratamiento de las colocaciones, puesto que este segmento fundamental para efectos de producción está incorporado a los ejemplos. Es muy difícil que un aprendiz inicial consiga identificarlo. En BugueÑo MiRAnda 2009b ya se llamaba la atención para este hecho cuando se comentaba que una tendencia de la lexicografía alemana es generar ejemplos polifuncionales que obedezcan a más de una función. En otras palabras, que sirvan, por ejemplo, de mecanismo de complementación a la paráfrasis o como prototipo de patrones sintácticos. La polifuncionalidad en un diccionario para aprendices iniciales es absolutamente desaconsejable si se considera la complejidad de este fenómeno lexicológico y su todavía insipiente competencia tanto en lo que se refiere a la lengua en sí como con relación a la interpretación de las informaciones que el diccionario ofrece. Ejemplos de colocaciones se pueden encontrar s.v. Fach $\mid$ hoch $\mid$ schu $\mid$ le "[...] die Fachhochschule besuchen"; s.v. Mi|ne "[...] eine Mine schließen, stillegen [...]"; s.v. sa|gen " [...] etwas laut,, leise, offen sagen [...]". 
BUGUEÑO MIRANDA, F. - A propósito de três diccionarios de DaF

Se escogió HuDuWtbDaF (2007) como diccionario de contraste con relación a LGwtbDaF (2015) y a DuWtbDaF(2015), diccionarios para aprendices avanzados, por considerarse que era el diccionario que mejor definía al público al que estaba destinado (aprendices de los niveles A1 a B1) ${ }^{23}$. Esa premisa se valida después del análisis realizado. Los errores de concepción macroestructural, tales como la no lematización de tokens referentes a las formas irregulares o el lugar discreto que ocupan los compuestos, se compensan con el ofrecimiento de paráfrasis de fácil comprensión, hecho este que cabe destacar. Sin embargo en el ámbito microestructural, si por una parte, el programa de constante de informaciones es mucho menos ambicioso que el de los otros dos diccionarios; por otra, el usuario no pierde el necesario Überblick que tanto se espera de este y de cualquier diccionario en realidad. La única nota realmente crítica se refiere a la desatención en la presentación y realce de las colocaciones, sobre todo, sabiendo que a estos patrones de combinatoria hay que prestarles la debida atención desde las fases iniciales del aprendizaje de cualquier lengua. Razón tenía Hausmann (2007) cuando afirmaba que "Apprendre le vocabulaire, c'est apprendre les collocations".

\section{Conclusiones}

En la parte introductoria de este trabajo se ha hecho alusión a la lexicografía de aprendices del inglés es pionera y guía, en cierta manera, de las obras de esta clase específica de diccionarios. No cabe duda de que la lexicografía de aprendices del alemán como un todo corresponde al estado del arte que presentan Jamada (2015) y Chi (2015).

En una síntesis crítico-contrastiva de los tres diccionarios entre sí afloran, sin embargo, ciertos aspectos que merecen una atención crítica.

En primer lugar, la clara diferencia en la definición macroestructural cuantitativa que presentan LGwtbDaF (2015) y DuWtbDaF (2015) demuestra que el cálculo de la densidad macroestructural sigue constituyendo un problema no resuelto en la lexicografía de aprendices, y en la lexicografía como un todo -dígase de paso-. La cuestión no es menor, si se considera que al estudiante de DaF hay que proporcionarle un espectro amplio de vocabulario, pero que corresponda a una previsión, aunque sea mínima, de sus necesidades. No hay como afirmar que LGwtbDaF (2015) presente una hinchazón

23 Cf. nota 7 del presente trabajo.

Pandaemonium, São Paulo, v. 22, n. 36, jan.-abr. 2019, p. 67-96 
BUGUEÑO MIRANDA, F. - A propósito de três diccionarios de DaF

macroestructural, pero se ha hecho empeño a que lemas como las formas irregulares de los verbos son perfectamente dispensables para un usuario avanzado de la lengua.

Cabe señalar que los trabajos citados apuntan claramente para una falta de parámetros que guíen la selección lemática. Aunque por una cuestión de espacio resultaba irrealizable para el presente trabajo, se plantea que un análisis comparativo de la densidad macroestructural entre LGwtbDaF (2015) y DuWtbDaF (2015) podría tal vez arrojar luces que permitan comprender mejor la diferencia de número de lemas entre los dos diccionarios.

Al otro extremo se encuentra HuDuWtbDaF (2007), obra que refleja mejor el desiderátum de Beißwenger y Körkel (2002). No obstante, habrá que reconocer que el conocimiento sobre esta estructura parcial de los diccionarios sigue siendo modesto.

Sin duda alguna es en el ámbito microestructural que los Lernerwörterbücher evaluados demuestran insoslayables cualidades. Todos, sin excepción, ofrecen una prolija descripción de la lengua en sus diversos niveles de organización. Cabe recordar en este contexto que una descripción exhaustiva de la lengua en uso no es una cualidad a priori en este tipo de obras lexicográficas, como lamentablemente lo demuestran los escasos diccionarios de aprendices de español, por ejemplo. Tampoco puede afirmarse que HuDuWtbDaF (2007) sea menos prolijo en esta tarea que LGwtbDaF (2015) y DuWtbDaF (2015), por el hecho de que el primero de estos diccionarios está diseñado para aprendices de ciclo inicial, mientras que los otros dos fueron concebidos pensando en el aprendiz avanzado. Considerando la diferencia de niveles, lo que se nota es una declarada recursividad en el caso de las dos obras destinadas a los aprendices avanzados. Ello, no obstante, no parece constituir una decisión que emane de una especial atención al público destinatario, sino que es un rasgo presente en los diccionarios semasiológicos generales del alemán, como DUW en sus varias ediciones. Tal característica no es ni $a$ priori ni a posteriori un demérito de tales obras, pero el día que los metalexicógrafos consigan elaborar instrumentos de evaluación para correlacionar densidad informativa con atención (o percepción en general, si se quiere) este será, sin duda alguna, uno de los primeros problemas que requerirán una atención inmediata, sobre todo para los niveles iniciales. El diseño de la microestructura de los tres diccionarios es gründlich.

No es posible soslayar una evaluación más precisa de las paráfrasis y de la descripción sintáctica de los verbos. Con relación al primer aspecto, se subrayó a lo largo del análisis que la definición continúa siendo un desafío para la lexicografía. Por ello los 
BUGUEÑO MIRANDA, F. - A propósito de três diccionarios de DaF

resultados concretos de las paráfrasis no siempre son completamente satisfactorios. No obstante, se nota el celo de los redactores por ofrecer alternativas para compensar los casos de paráfrasis poco claras. La sustitución ostensiva es un camino. En lo tocante a los verbos, es esta una categoría en que la recursividad se hace patente para alivio del aprendiz.

Para el final hemos dejado la función de producción textual. Esta solo puede ser evaluada en el contexto de un diccionario de orientación semasiológica. La sinonimia y la antonimia se ofrecen necesariamente como soluciones paliativas en la ausencia de un diccionario onomasiológico stricto sensu concebido ex professo para aprendices de DaF. Hasta ahora tal obra no existe. Tan importante como estas dos magnitudes para la producción son las combinatorias léxicas. Si lamentablemente HuDuWtbDaF (2007) trata implícitamente las colocaciones, los otros dos diccionarios son ricos en esta clase de informaciones.

\section{Referencias bibliográficas}

Diccionarios analizados

DuWtbDaF. Duden Deutsch als Fremdsprache. Standardwörterbuch. Berlin: Dudenverlag, 2015. HuDuWtbDaF. Hueber Wörterbuch Deutsch als Fremdsprache. Ismaning/Mannheim: Hueber/Duden, 2007.

LGwtbDaF. Langenscheidt Großwörterbuch Deutsch als Fremdsprache. München/Wien: Langenscheidt, 2015.

Demás bibliografía

BEIBWENGER, Michael; KÖRKEL, Boris. Die Lemmaselektion im de Gruyter Wörterbuch Deutsch als Fremdsprache. In: WIEGAND, Herrbert Ernst (Hrsg.) Perspektiven der pädagogischen Lexikographie des Deutschen II. Untersuchungen anhand des ,de Gruyter Gruyter Wörterbuch Deutsch als Fremdsprache“. Tübingen: Niemeyer, 2002, p. 393-412. Disponível em: <http://www.michael-beisswenger.de/pub/bk-lemma.pdf2> Acesso em 23.12.2017.

BIELÍŃSKA, Monika. Lexikographische Metatexte: eine Untersuchung nichtintegrierter Außentexte in einsprachigen Wörterbüchern des Deutschen als Fremdsprache. Frankfurt am Main: Peter Lang (= Danziger Beiträger zur Germanistik 32), 2010.

BorbA, Laura Campos de; Bugueño Miranda, Félix. Análise de cinco dicionários semasiológicos de língua espanhola: a correlação entre o Front Matter e a Macro e a Microestrutura. Extensio, v. 9, n. 14, 2012, p. 32-43. Disponível em: <https://periodicos.ufsc.br/index.php/extensio/article/view/1807-0221.2012v9n14p32/ 24297> Acesso em 11.12.2017. 
BUGUEÑO MIRANDA, F. - A propósito de três diccionarios de DaF

BRAY, Laurent. Consultabilité et lisibilité du dictionnaire: aspects formels. In: HAUSMANN, Franz et al. (Hrsg.). Wörterbücher, Dictionaries, Dictionnaires. An international Encyclopedia of Lexicography I. Berlin/New York: de Gruyter, 1989, p. 135-146.

BugueÑo Miranda, Félix. As soluções polissêmicas e homonímicas em dicionários semasiológicos. Revista Trama, v. 12, n. 24, 2016, p. 121-153. Disponível em: <http://erevista.unioeste.br/index.php/trama/article/view/11424/9415> Acesso em 27.12.2017.

. Para uma taxonomia de paráfrases explanatórias. Alfa, v. 53, n. 1, 2009a, p. 243-260. Disponível em: <http://seer.fclar.unesp.br/alfa/article/view/1686/1367> Acesso em 16.12.2017.

. Sobre a microestrutura de dicionários semasiológicos do alemão. Contingentia, v. 4, n. 2, 2009b, p. 60-72. Disponível em: <http://www.seer.ufrgs.br/contingentia/article/view/11414/6777F> Acesso em 09.01.2018.

. O que é macroestrutura no dicionário de lingua?. In: IsQueRdo, Aparecida Negri; Alves, Ieda Maria. (Org.). As ciências do Léxico III: Lexicologia, lexicografia e terminologia. São Paulo: Humanitas, 2007, p. 261-272.

BugueÑo Miranda, Félix; ReOlon JARDiM, Carolina. Os learner's dictionaries do inglês e os Lernwörterbücher do alemão: uma simples transposição de nomes? Contingentia, v. 5, n. 1, 2010, p. 41-67. Disponível em: <http://seer.ufrgs.br/index.php/contingentia/article/view/13319/7614> Acesso em 03.01.2018)

Bugueño MiRanda, Félix; ZanatTa, Flávia. Procedimentos medioestruturais em dicionários semasiológicos de língua portuguesa. Lusorama, v. 83-84, 2010, p. 80-97.

BUGUEÑo MiRANDA, Félix; FARIAS, Virgínia. Los subsidios de tres teorías semánticas para la generación de definiciones lexicográficas. Revista de Filología de la Universidad de la Laguna, n. 31, 2013, p. 19-34.

Bugueño Miranda, Félix; Farias, Virgínia. Desenho da macroestrutura de um dicionário escolar de língua portuguesa. In: XATARA, Cláudia; BeVILACQuA, Cleci; Humblé, Philippe (Org.). Lexicografia Pedagógica: Pesquisas e Perspectivas. Florianópolis: UFSC/NUT, 2008, p. 129-167.

CHI, Amy. Researching Pedagogical Lexicography. In: JACKSON, Howard (Ed.). The Bloomsbury Companion to Lexicography. London/New York: Bloomsbury, 2015, p. 165-187.

COSERIU, Eugenio. Einführung in die Allgemeine Sprachwissenschaft. Tübingen: Francke, 1992.

DuGram. Duden. Die Grammatik. Mannheim: Bibliographisches Institut, 2009.

DLexFr. TOURnier, Nicole; TOURniER, Jean. Dictionnaire de lexicologie française. Paris: Ellipses, 2017.

DUW. Deutsches Universalwörterbuch. Mannheim: Dudenverlag, 1999.

ENGELBERG, Stefan; LEMNITZER, Lothar. Lexikographie und Wörterbuchbenutzung. Tübingen: Stauffenburg, 2004.

FARIAS, Virginia. O exemplo como informação discreta e discriminante em dicionários semasiológicos de língua portuguesa. Alfa, v. 52, n. 1, 2008, p. 101-122. Disponível em: <http://seer.fclar.unesp.br/alfa/article/view/1469/1174> Acesso em 20.12.2017.

GER. Gemeinsamer europäischer Referenzrahmen für Sprachen. Berlin: Langenscheidt, 2001. Disponível em: 〈http://student.unifr.ch/pluriling/assets/files/Referenzrahmen2001.pdf> Acesso em 19.12.2017.

HARTMANN, R.R.K; JAMES, Gregory. Dictionary of lexicography. London: Routledge, 2001.

HAUSMANN, Franz Joseph. Apprendre le vocabulaire, c'est apprendre collocations. In: HaUSMANN, Franz Joseph. Collocations, phrasélogie, lexicographie. Études 1977-2007 et Bibliographie. Aachen: Shaker Verlag, 2007, p. 49-61.

Herbst, Thomas; KLOTZ, Michael. Lexikographie. Paderborn, Ferdinand Schöningh, 2003.

Pandaemonium, São Paulo, v. 22, n. 36, jan.-abr. 2019, p. 67-96 
BUGUEÑO MIRANDA, F. - A propósito de três diccionarios de DaF

JACKSON, Howard. Lexicography. An Introduction. London: Routledge, 2002.

JAMADA, Shigeru. Monolingual Learner's dictionaries - Where now? In: JACKSON, Howard (Ed.). The Bloomsbury Companion to Lexicography. London/New York: Bloomsbury, 2015, p. 188-212.

KILGARRIFF, Adam. I don't believe in Word Senses. In: FonTANELle, Thierry (Ed.). Practical Lexicography. A reader. Oxford: OUP, 2008, p. 135-151.

KISPÁL, Tamás. Lexikographie der Jugendsprache in neueren deutschen Lernerwörterbüchern. In: BergerovÁ, Hana; SCHMIDT, Marek; SCHUPPENER, Georg. Aussiger Beiträge. Germanistische Schriftenreihe aus Forschung und Lehre 7. Wien: Praesens, 2013, p. 87101.

KOEPPEL, Rolf. Deutsch als Fremdsprache. Spracherwerblich reklektierte Unterrichtspraxis. Baltmannsweiler: Schneider Verlag, 2013.

MLSpr. GLÜCK, Helmut (Hrsg.). Metzler Lexikon Sprache. Stuttgart: J.B.Metzler, 2010.

MÖHRING, Jupp; WALLNER, Franziska. Wortschatzlisten auf dem Prüfstand. In: BERGEROVÁ, Hana; SCHMIDT, Marek; SCHUPPENER, Georg. Aussiger Beiträge. Germanistische Schriftenreihe aus Forschung und Lehre 7. Wien: Praesens, 2013, p. 119-133.

NATION, I.S.P. Learning vocabulary in another language. Cambridge, CUP, 2006.

PROFILE DeUtSch. Glaboniat, Manuela et al. Profile Deutsch. Berlin/München/Wien/Zürich/New York: Langenscheidt, 2005.

RoTHENHÖFER, Andreas. New developments in learner's dictionaries II: German. In: Gouws, Rufus et al. (Eds.). Wörterbücher, Dictionaries, Dictionnaires. An international Encyclopedia of Lexicography. Supplementary volume. Berlin: de Gruyter, 2013, p. 414425.

RUNDELL, Michael. Recent trends in English Pedagogical Lexicography. In: FonTENELLE, Thierry (Ed.). Practical Lexicography. A reader. Oxford: OUP, 2008, p. 221-243.

SCHLAEFER, Michael. Lexikologie und Lexikographie. Eine Einführung am Besipiel deutscher Wörterbücher. Berlin, Erich Schmidt Verlag, 2009.

RUNTE, Maren. Lernerlexikographie und Wortschatzerwerb. Berlin: de Gruyter, 2015.

STÄDTLER, Thomas. Wissenschaftliche Lexikographie im deutschsprachigen Raum. Heidelberg: Carl Winter Verlag, 2003.

THUMB, Jenny. Dictionary look-up strategies and the bilingualized learner's dictionary. Tübingen: Niemeyer, 2004.

VerLinde, Serge; BINON, Jean. Pedagogical Lexicography Revisited. In: Bergenhotz, Henning; NIElSEN, Sandro; TARP, Sven (Ed.). Lexicography at the Crossroad. Dictionaries and Encyclopedias Today, Lexicographical Tools Tomorrow. Frankfurt: Peter Lang, 2009, p. 69-89.

WANZECK, Christiane. Lexikologie. Beschreibung von Wort und Wortschatz im Deutschen. Göttingen: Vandenhoeck \& Ruprecht, 2010.

WtbLBeg. ULRICH, Winfried. Wörterbuch Linguistische(r) Begriffe. Berlin/Stuttgart: Gebrüder Borntraeger, 2002.

YAMADA, Shigeru. Monolingual Learner's Dictionaries - Where Now? In: JACKSON, Howard (Ed.). The Bloomsbury Companion to Lexicography. London/New York: Bloomsbury, 2015, p. 188-212.

Zgusta, Ladisłav. Manual of Lexicography. Praha: Akademie, 1971.

Recebido em 28 de março de 2018 Aceito em 01 de julho de 2018

Pandaemonium, São Paulo, v. 22, n. 36, jan.-abr. 2019, p. 67-96 